\title{
Decreased expression of the long non-coding RNA FENDRR is associated with poor prognosis in gastric cancer and FENDRR regulates gastric cancer cell metastasis by affecting fibronectin1 expression
}


Er-bao Zhang ${ }^{3}$, Rong Kong ${ }^{3}$, Wei De ${ }^{3}$ and Yong-qian Shu ${ }^{{ }^{*}}$

\begin{abstract}
Background: FENDRR is a long non-coding RNAs (InCRNA) that binds to polycomb repressive complexe 2 (PRC2) to epigenetically regulate the expression of its target gene. The clinical role of FENDRR in carcinomas remains yet to be found.

Method: Real-time polymerase chain reaction (PCR) was used to examine FENDRR expression in gastric cancer cell lines/tissues compared with normal epithelial cells/adjacent non-tumorous tissues. Cell proliferation assays, Wound healing assays, and in vitro and in vivo invasion and migration assays were performed to detect the biological effects of FENDRR in gastric cancer cells. Real-time PCR, western-blot and immunohistochemistry were used to evaluate the mRNA and protein expression of fibronectin1 (FN1). Secreted matrix metalloproteinase (MMP) activities were detected and characterized using gelatin zymography assay.

Results: FENDRR was downregulated in gastric cancer cell lines and cancerous tissues, as compared with normal gastric epithelial cells and adjacent noncancerous tissue samples. Low FENDRR expression was correlated with deeper tumor invasion $(p<0.001)$, higher tumor stage $(p=0.001)$, and lymphatic metastasis $(p=0.007)$. Univariate and multivariate analyses indicated that low FENDRR expression predicted poor prognosis. Histone deacetylation was involved in the downregulation of FENDRR in gastric cancer cells. FENDER overexpression suppressed invasion and migration by gastric cancer cells in vitro, by downregulating FN1 and MMP2/MMP9 expression.
\end{abstract}

Conclusion: Low expression of the IncRNA FENDRR occurs in gastric cancer and is associated with poor prognosis. Thus, FENDRR plays an important role in the progression and metastasis of gastric cancer.

Keywords: Fibronectin1, FENDRR, Metastasis, Prognosis, Gastric cancer

\footnotetext{
* Correspondence: yongqian_shu@163.com

${ }^{\dagger}$ Equal contributors

'Department of Oncology, the First Affiliated Hospital of Nanjing Medical University, No. 300 Guangzhou Road, Nanjing 210029, Jiangsu Province,

People's Republic of China

Full list of author information is available at the end of the article
} 


\section{Background}

Gastric cancer is the fourth most common malignancy in the world and is the second most frequent cause of cancer-related deaths worldwide, with particularly high incidence in East Asia [1,2]. Although gastric cancer is curable if detected early, most patients are diagnosed in the advanced stage and have poor prognosis [3]. Tumor invasion and metastasis are critical steps in determining aggressive tumor phenotype and they also account for many cancer-related deaths [4]. The clinical stage, based on the TNM classification system, at the time of diagnosis is currently the most important prognostic factor, and the molecular mechanism involved in progression and metastasis of gastric cancer remains unclear [5]. Thus, novel findings on prognosis factors that are associated with gastric cancer progression and metastasis would be of great clinical relevance.

Apart from about $2 \%$ protein-coding genes, the vast majority of the human genome is made up of non-coding RNAs (ncRNAs), indicating that ncRNAs could play significant regulatory roles in complex organisms [6]. These noncoding regions are interspersed throughout genomic DNA. One subcategory of these transcripts, called long noncoding RNAs (lncRNAs), comprises ncRNAs that are more than 200 nucleotides in length. It is known that lncRNAs are widely transcribed in the genome, but our understanding of their functions is limited. Many studies have revealed that the deregulated expression of IncRNAs plays a functional role in a variety of disease states $[7,8]$. In addition, recent reports have showed that some lncRNAs exhibit distinct gene-expression patterns and play significant roles during cellular development in various types of carcinomas [9-13]. However, the overall pathophysiological contributions of lncRNAs to gastric carcinoma remain largely obscure. Functional lncRNAs can be used for cancer diagnosis and prognosis, and serve as potential therapeutic targets; thus, lncRNAs can be considered as a new diagnostic and therapeutic gold mine in cancer [14].

The FENDRR gene is 3099nts in length, located at chr3q13.31, and consists of four exons. It is an lncRNA that is essential for proper heart and body wall development in mouse [15]. FENDRR can bind to both polycomb repressive complexe 2 (PRC2) and Trithorax group/MLL protein complexes (TrxG/MLL), which play pivotal roles in the control of chromatin structure and gene activity $[16,17]$. HOTAIR, which is one of the few well-studied lncRNAs, plays a significant role in tumor progression by regulation of oncogene or tumor suppressor gene expression through binding to PRC2 [18]. Considering the role of HOTAIR, we hypothesized that FENDRR, another PRC2-binding lncRNA, may also be involved in tumorigenesis.

In this study, we found that FENDRR expression was reduced in gastric cancer tissues and cell lines. Low expression of FENDRR was associated with clinicopathological characteristics and poor prognosis in gastric cancer patients. Histone deacetylation contributed to the decreased expression of FENDRR in gastric cancer cells. Ectopic expression of FENDRR in gastric cells significantly inhibited cell migration and invasion. Conversely, depletion of FENDRR promoted these activities. Moreover, we found that fibronectin1 (FN1) and secreted matrix metalloproteinase (MMP) 2/ (MMP) 9 were involved in the FENDRR-mediated inhibition of cell migration and invasion. These results suggest FENDRR plays a significant role in the progression and metastasis of gastric cancer and could be used as a new therapeutic target.

\section{Results}

FENDRR expression was downregulated in gastric cancer tissues and cell lines, and histone deacetylation was involved in the downregulation of FENDRR

FENDRR expression levels were investigated in 158 paired gastric cancer samples and adjacent histologically normal tissues using quantitative polymerase chain reaction (qPCR) assays. FENDRR expression was significantly lower in tumor tissues than in the adjacent normal tissues $(\mathrm{p}<0.05$; Figure 1A). Reverse transcription (RT)-qPCR assays were further developed to quantify FENDRR in gastric cancer cell lines, including MGC803, BGC823, MKN28, MKN45 and SGC7901, and in the normal gastric epithelium cell line GES1. Significantly lower expression of FENDRR was found in MKN28 $(\mathrm{p}=0.031)$, MKN45 $(\mathrm{p}=0.041)$ and MGC803 $(\mathrm{p}=0.035)$ than in GES-1, but there was no significant difference for BGC823 and SGC7901 (Figure 1B).

Next, we investigated the mechanisms controlling the tissue-specific expression of FENDRR. We analyzed the promoter and the first exon region of FENDRR, and found that there were two $\mathrm{CpG}$ islands. However, FENDRR expression was not changed after treatment with the DNA methyhransferase (DNMT) inhibitor 5-azacytidine (5-aza-C), indicating that DNA methylation contribution little to FENDRR expression (data not shown). Histone protein modification was thought to play an important role in the transcription of lncRNAs; however, the knockdown of two core subunits of PRC2 (SUZ12 and EZH2) had no influence on FENDRR expression (data not shown). Interestingly, FENDRR expression was induced in MGC803 and BGC823 cells after treatment with the histone deacetylase (HDAC) inhibitor trichostatin A (TSA) (Figure 1C). We sought to determine whether the inhibition of FENDRR was mediated by HDACs. Specific anti-HDAC1 and HDAC3 small interfering RNAs (siRNAs) were transfected into GC (gastric cancer) cells, and HDAC1 and HDAC3 expression was significantly decreased (Additional file 1: Figure S1). The expression levels of FENDRR were 


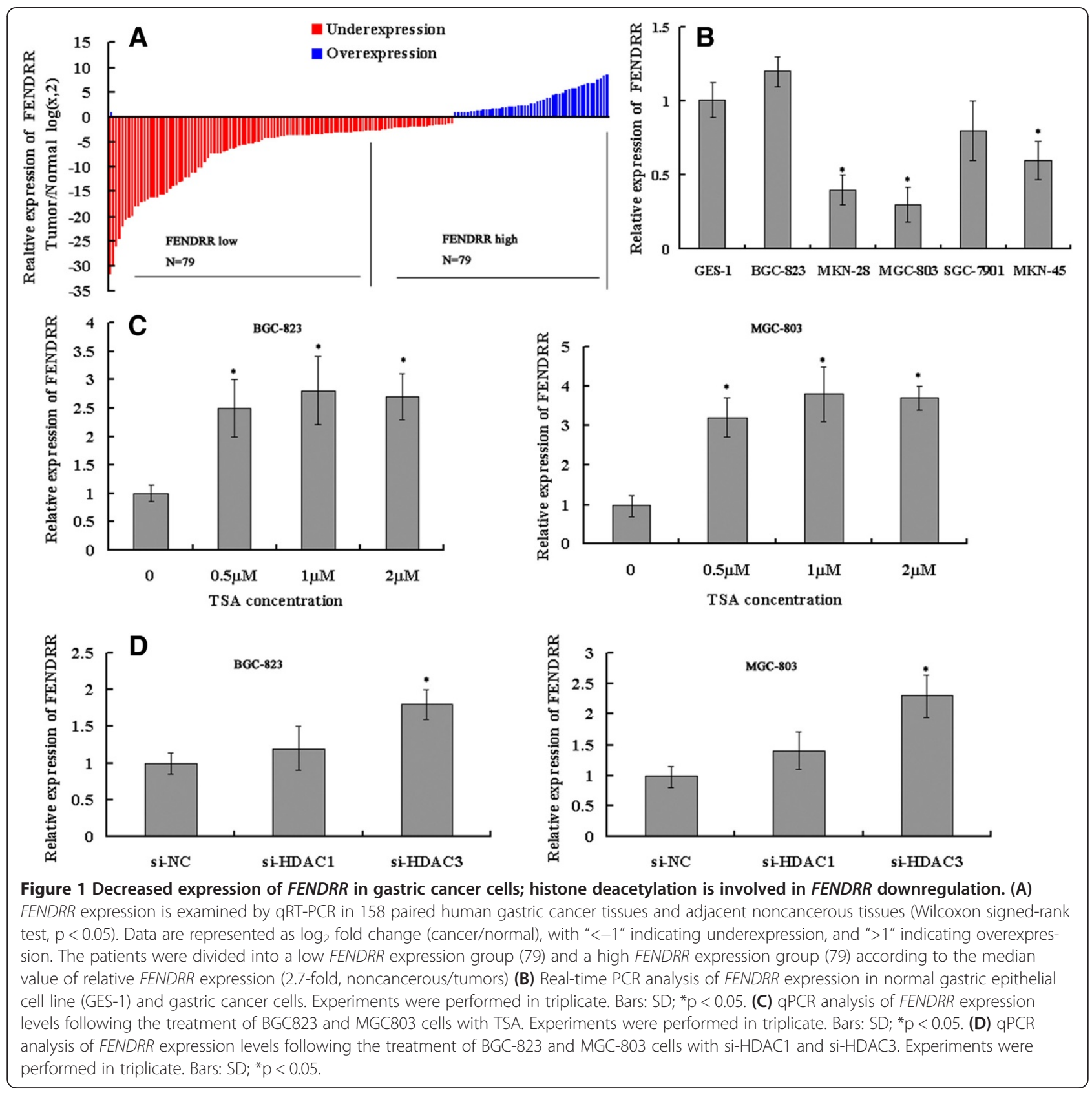

significantly upregulated in cells transfected with siHDAC3 but not in those transfected with scrambled siRNA or si-HDAC1 (Figure 1D). These data indicate that the HDAC3 knockdown-mediated increase in FENDRR expression may be due to the inhibition of HDAC3 enzymatic activity.

\section{FENDRR expression and clinicopathologic factors in gastric cancer}

To assess the correlation of FENDRR expression with clinicopathologic data, the expression levels of FENDRR in tumor tissues were categorized as low or high in relation to the median value of relative FENDRR expression (2.7-fold, noncancerous/tumors). Clinicopathologic factors were analyzed in the high and low FENDRR expression groups. As shown in Table 1 , the low FENDRR group $(\mathrm{n}=79)$ showed greater invasion depth $(\mathrm{p}<0.001)$, higher tumor stage $(\mathrm{p}=$ $0.001)$, and more frequent lymphatic metastasis $(\mathrm{p}=0.007)$ than the high FENDRR expression group $(\mathrm{n}=79)$. However, there was no significant correlation between FENDRR expression and other clinicopathologic features such as age, sex, tumor location, tumor size, histologic grade, and distant metastasis $(\mathrm{p}>0.05)$. Clinical data of all patients is shown in Additional file 2: Table S2. 
Table 1 Correlation between FENDRR expression and clinicopathological characteristics of gastric cancer

\begin{tabular}{|c|c|c|c|}
\hline \multirow[t]{2}{*}{ Clinical parameter } & \multicolumn{2}{|l|}{ FENDRR } & \multirow{2}{*}{$\begin{array}{l}\text { Chi-squared } \\
\text { test P-value }\end{array}$} \\
\hline & $\begin{array}{l}\text { High No. cases } \\
(n=79)\end{array}$ & $\begin{array}{l}\text { Low No. cases } \\
(n=79)\end{array}$ & \\
\hline Age (years) & & & 0.426 \\
\hline$<50$ & 35 & 40 & \\
\hline$>50$ & 44 & 39 & \\
\hline Gender & & & 0.255 \\
\hline Male & 44 & 51 & \\
\hline Female & 35 & 28 & \\
\hline Location & & & 0.452 \\
\hline Distal & 31 & 37 & \\
\hline Middle & 28 & 28 & \\
\hline Proximal & 20 & 14 & \\
\hline Size & & & 0.152 \\
\hline$>5 \mathrm{~cm}$ & 37 & 46 & \\
\hline$<5 \mathrm{~cm}$ & 42 & 33 & \\
\hline $\begin{array}{l}\text { Histologic } \\
\text { differentiation }\end{array}$ & & & 0.171 \\
\hline Well & 4 & 6 & \\
\hline Moderately & 34 & 21 & \\
\hline Poorly & 34 & 41 & \\
\hline Undifferentiated & 7 & 11 & \\
\hline Invasion depth & & & $<0.001^{*}$ \\
\hline $\mathrm{T} 1$ & 30 & 6 & \\
\hline $\mathrm{T} 2$ & 28 & 13 & \\
\hline T3 & 11 & 34 & \\
\hline $\mathrm{T} 4$ & 10 & 26 & \\
\hline TNM Stages & & & $0.001^{*}$ \\
\hline । & 22 & 8 & \\
\hline$\|$ & 32 & 23 & \\
\hline III & 16 & 39 & \\
\hline IV & 9 & 9 & \\
\hline Lymphatic metastasis & & & $0.007^{*}$ \\
\hline Yes & 33 & 50 & \\
\hline No & 46 & 29 & \\
\hline Regional lymph nodes & & & $<0.001^{*}$ \\
\hline PNO & 47 & 28 & \\
\hline PN1 & 15 & 7 & \\
\hline PN2 & 10 & 27 & \\
\hline PN3 & 7 & 17 & \\
\hline Distant metastasis & & & 0.797 \\
\hline Yes & 8 & 9 & \\
\hline No & 71 & 70 & \\
\hline
\end{tabular}

*Overall $p<0.05$.
Low FENDRR expression is associated with poor prognosis of patients with gastric cancer

Kaplan-Meier analysis and log-rank test were used to evaluate the effects of FENDRR expression and the clinicopathological characteristics on disease-free survival (DFS) and overall survival (OS). The results showed that patients in the low FENDRR expression group had a higher recurrence rate (median DFS: 14 months) and much shorter overall survival (median OS: 19 months) than those in the high FENDRR expression group (median DFS: 25 months; median OS: 35 months; $\mathrm{p}=0.006$ and 0.008 , respectively; Figure 2A,E).The 3-year DFS and OS were $29.2 \%$ and $36.7 \%$,respectively, in the low FENDRR expression group, and $39.2 \%$ and $46.2 \%$, respectively in the high FENDRR expression group. Moreover, the expression of FENDRR was strongly correlated with DFS and OS in the advanced clinical stages (stage III and IV; Figure 2D,G,H). However, in patients in clinical stages I and II, no significant difference in DFS and OS were found between those with low and high FENDRR expression (Figure 2B,F). Univariate analyses of clinical variables considered as potential predictors of survival are shown in Table 2. Further analysis in a multivariate Cox proportional hazards model showed that FENDRR expression, together with TNM stage, was strongly associated with OS. Furthermore, FENDRR expression was also significantly correlated with DFS in our study cohort. FENDRR expression was an independent prognostic indicator of DFS (hazard ratio $[\mathrm{HR}]=0.555$; 95\% confidence interval $[\mathrm{CI}], 0.344-0.897 ; \mathrm{p}=0.016)$ and OS (HR $=0.569 ; 95 \%$ CI, $0.321-0.960 ; \mathrm{p}=0.042)$ in patients with gastric cancer (Table 2).

\section{FENDRR exhibits an insignificant effect on gastric cancer} cell proliferation, but represses gastric cancer cell migration and invasion in vitro

We further evaluated the role of FENDRR in cell proliferation, migration and invasion. FENDRR was over-expressed by transfecting pcDNA3.1-FENDRR vector into MGC803 cell line, which has a relatively low level of FENDRR expression. In addition, FENDRR was depleted in BGC823 cells, which exhibit a higher expression of FENDRR. The ectopic expression and knockdown of FENDRR in cells was confirmed by qRT-PCR. (Figure 3A).However, none of MTT assays and colony formation assays detected a significant proliferative effect of FENDRR in either the MGC803 or the BGC823 cell line (Figure 3B,C). Subsequently, we observed the effect on cell migration and invasion. As shown in Figure 3D, MGC803 cells, which have a naturally low FENDRR expression, when transfected for over-expression of FENDRR exhibited a notably lower scratch closure rate (migration inhibition) than observed in controls infected with empty vector. Moreover, BGC823 cells, which have a naturally high FENDRR expression, after 


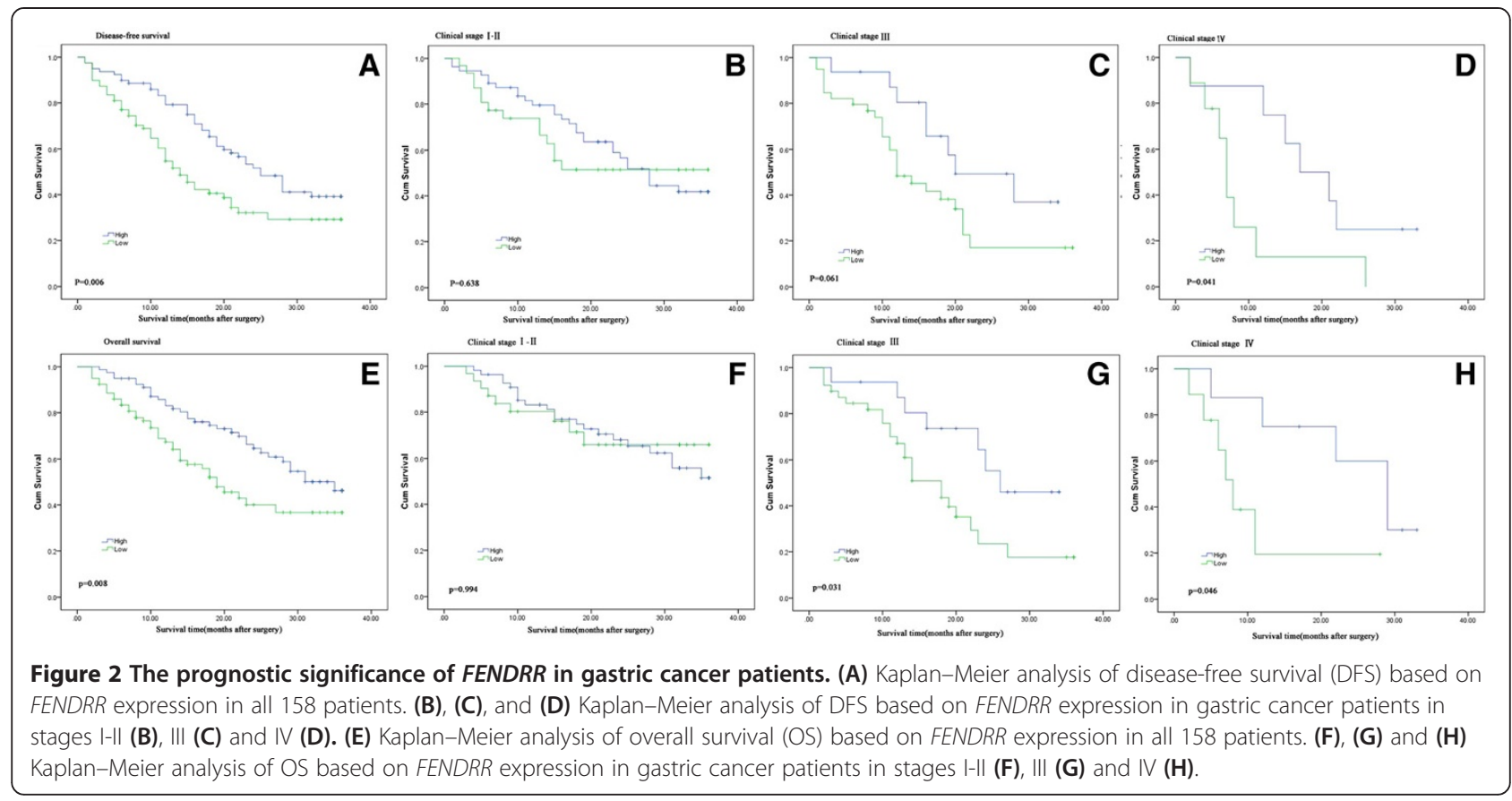

knockdown of FENDRR, displayed a higher scratch closure rate (migration promotion) than control cells. Furthermore, cell motility was also measured using migration and invasion assay. Compared with the control cells, FENDRRoverexpressing MGC803 cells showed markedly repressed migration and invasion ability $(\mathrm{p}<0.05$; Figure $3 \mathrm{E}, \mathrm{F})$. Likewise, knockdown of FENDRR significantly stimulated migration and invasion of BGC823 cells $(\mathrm{p}<0.05$, Figure $3 \mathrm{E}$, F). These findings indicate that FENDRR may be closely associated with invasion and migration of gastric cancer cell lines.

\section{FENDRR suppresses GC cell metastasis in vivo}

To validate the effects of FENDRR on the metastasis of GC cells in vivo, MGC803 cells stably transfected with pcDNA-FENDRR were injected into nude mice. Metastatic nodules on the surface of lungs were counted after 7 weeks. Ectopic overexpression of FENDRR reduced the number of metastatic nodules compared with those in the control group (Figure 3G). This difference was further confirmed following examination of the entire lungs, and through hematoxylin and eosin (HE) staining of lung sections (Figure $3 \mathrm{H}$ ). Our in vivo data complemented the results of functional in vitro studies involving FENDRR.

\section{Downregulated expression of FN1 and MMPs is involved in the FENDRR-mediated inhibition of gastric cancer cell metastasis}

To explore the molecular mechanisms by which FENDRR contributes to the phenotypes of gastric cancer cells, we investigated potential targets involved in tumor invasion and metastasis. First, by using qRT-PCR, we detected the host gene FOXF1, which plays an important role in cancer cell invasion and migration [19], in FENDRR-overexpressing cells, FENDRR-knockdown cells, and control cells. However, no changed expression was found (data not shown). The result demonstrated that FENDRR may not exert its functions by regulating its host gene. Cell adhesion molecules are implicated in invasion and metastasis in various cancers. Therefore, we performed qRT-PCR to detect the expression of cell adhesion molecules that have been confirmed to be involved in tumor invasion and metastasis (such as, fibronectin1 [FN1], integrin, CD44, ICAM-1, E-cadherin, N-cadherin and Vimentin). Interestingly, fibronectin1 (FN1) was found to be significantly altered among them. When FENDRR was overexpressed or blocked, FN1 mRNA was diminished by $70 \%$ or elevated 2.0-fold, respectively, compared to the control groups (Figure 4A,B). Reports have shown that FN1 is associated with tumor migration and invasion [20]. Therefore, we determined the expression of the FN1 protein by performing western blot analysis. The FN1 protein level was also reduced by approximately $60 \%$ in MGC803 cells transfected with pcDNA-FENDRR (Figure 4C) and was elevated 2.0-fold after transfection of the cells with siFENDRR (Figure 4D) compared to the respective controls. These data show that FN1 is negatively regulated by FENDRR at the mRNA and protein levels. MMPs are well known to contribute to cell invasion and metastasis in human carcinomas. We also found that the induction of FENDRR potently reduced the activity of MMP2/MMP9 
Table 2 Univariate and multivariate Cox regression analyses FENDRR for DFS or OS of patients in study cohort $(n=158)$

\begin{tabular}{|c|c|c|c|c|c|c|}
\hline \multirow[t]{2}{*}{ Variables } & \multicolumn{3}{|c|}{ DFS } & \multicolumn{3}{|c|}{ OS } \\
\hline & $\overline{\mathrm{HR}}$ & $95 \% \mathrm{Cl}$ & $\overline{p \text { value }}$ & $\overline{\mathrm{HR}}$ & $95 \% \mathrm{Cl}$ & $\mathrm{p}$ value \\
\hline \multicolumn{7}{|l|}{ Univariate analysis } \\
\hline Age ( $<50$ years vs. $>50$ years $)$ & 0.935 & $0.618-1.415$ & 0.752 & 0.954 & $0.599-1.520$ & 0.843 \\
\hline Gender (male vs. female) & 0.805 & $0.525-1.233$ & 0.318 & 0.771 & $0.475-1.252$ & 0.293 \\
\hline Location (Distal vs. Middle + Proximal) & 0.827 & $0.546-1.254$ & 0.372 & 0.714 & $0.448-1.138$ & 0.157 \\
\hline Tumor size ( $>5$ cm vs. $<5 \mathrm{~cm}$ ) & 1.392 & $0.917-2.113$ & 0.120 & 1.236 & $0.975-1.565$ & 0.079 \\
\hline Histologic differentiation(Well + Moderately vs. Poorly + Undifferentiated) & 1.264 & $0.826-1.933$ & 0.281 & 1.518 & $0.932-2.474$ & 0.094 \\
\hline Invasion depth (T1 + T2 vs.T3 + T4) & 1.306 & $0.863-1.978$ & 0.207 & 1.323 & $0.829-2.111$ & 0.240 \\
\hline TNM stage (I + II vs. III + IV) & 0.470 & $0.308-0.717$ & $<0.001^{*}$ & 0.405 & $0.251-0.653$ & $<0.001^{*}$ \\
\hline Lymphatic metastasis (No vs. Yes) & 0.617 & $0.404-0.924$ & $0.025^{*}$ & 0.624 & $0.389-1.000$ & $0.05^{*}$ \\
\hline Regional lymph nodes (PN2+ PN3 vs. PN0+ PN1) & 0.626 & $0.410-0.954$ & $0.029^{*}$ & 0.570 & $0.356-0.912$ & $0.019^{*}$ \\
\hline Distant metastasis (No vs. Yes) & 0.493 & $0.279-0.874$ & $0.015^{*}$ & 0.523 & $0.275-0.996$ & $0.049^{*}$ \\
\hline Expression of FENDRR (High vs. Low) & 0.563 & $0.370-0.856$ & $0.007^{*}$ & 0.539 & $0.337-0.862$ & $0.010^{*}$ \\
\hline \multicolumn{7}{|l|}{ Multivariate analysis } \\
\hline TNM stage $(I+||$ vs. III + IV) & 0.566 & $0.333-0.962$ & $0.035^{*}$ & 0.458 & $0.253-0.828$ & $0.010^{*}$ \\
\hline Lymphatic metastasis (No vs. Yes) & 0.740 & $0.408-1.342$ & 0.322 & 0.913 & $0.458-1.822$ & 0.797 \\
\hline Regional lymph nodes (PN0+ PN1vs. PN2+ PN3) & 0.658 & $0.338-1.283$ & 0.220 & 0.779 & $0.363-1.674$ & 0.522 \\
\hline Distant metastasis (No vs. Yes) & 0.577 & $0.298-1.117$ & 0.103 & 0.672 & $0.320-1.411$ & 0.294 \\
\hline Expression of FENDRR (High vs. Low) & 0.555 & $0.344-0.897$ & $0.016^{*}$ & 0.569 & $0.321-0.960$ & $0.042^{*}$ \\
\hline
\end{tabular}

*Overall $p<0.05$.

in GC cells, and inhibition of FENDRR contributed to the activation of MMP2/MMP9, corroborating our earlier finding that FENDRR protected against gastric cancer cell metastasis (Figure 4E,F).

\section{FN1 knockdown also suppresses cellular migration and} invasion in vitro

FN1 has been implicated in invasion and metastasis in diverse cancer; however, its role in gastric cancer is less well studied.. To determine the influence of FN1 on migration and invasion by gastric cancer cells, FN1 expression was knocked down by a siRNA targeting FN1 in MGC803 and BGC823 cells. Western blot and qRT-PCR analyses showed that transfection of gastric cancer cells effectively inhibited approximately $85 \%$ of FN1 expression at the mRNA level and $60 \%$ at the protein level, as compared to the expression in the control groups (Additional file 3: Figure S2A,S2B). Next, transwell assays examining cell migration and invasion were performed. On average, fewer si-FN1-transfected MGC803 and BGC823 cells than control cells were seen to migrate and invade (Figure 5A,B). A gelatin zymography assay revealed that depletion of FN1 also reduced MMP2/MMP9 expression (Figure 5C), which is consistent with previous reports $[21,22]$. These results indicate that FN1 knockdown suppresses cell migration and invasion in gastric carcinoma cells, partially via downregulation of MMP2 and MMP9.

Inhibition of FN1 is potentially involved in the tumorsuppressor function of FENDRR

To investigate whether FN1 was involved in the FENDRR-induced decrease in gastric cancer cell metastasis, we carried out rescue experiments. After transfection with si-FN1, BGC823 cells were cotransfected with si-FENDRR. We found that cotransfection of si-FN1 could rescue the upregulated expression of FN1 protein and MMP2/MMP9 induced by the depletion of FENDRR (Figure 5D,E). Moreover, migration and invasion assays indicated that the cotransfection could partially rescue siFENDRR-promoted metastasis in BGC823 cells (Figure 5F). These data indicated that FENDRR inhibits gastric cancer cell migration and invasion partly through the downregulation of FN1 expression.

\section{Inverse relationship between the expression of FN1 and FENDRR}

To assess the relationship between FN1 and FENDRR expression in gastric cancer, we examined FN1 expression by qPCR and immunohistochemistry in 40 paired gastric cancer tissues and in 5 gastric cancer cell lines. The results showed that the mRNA levels of FN1 were 


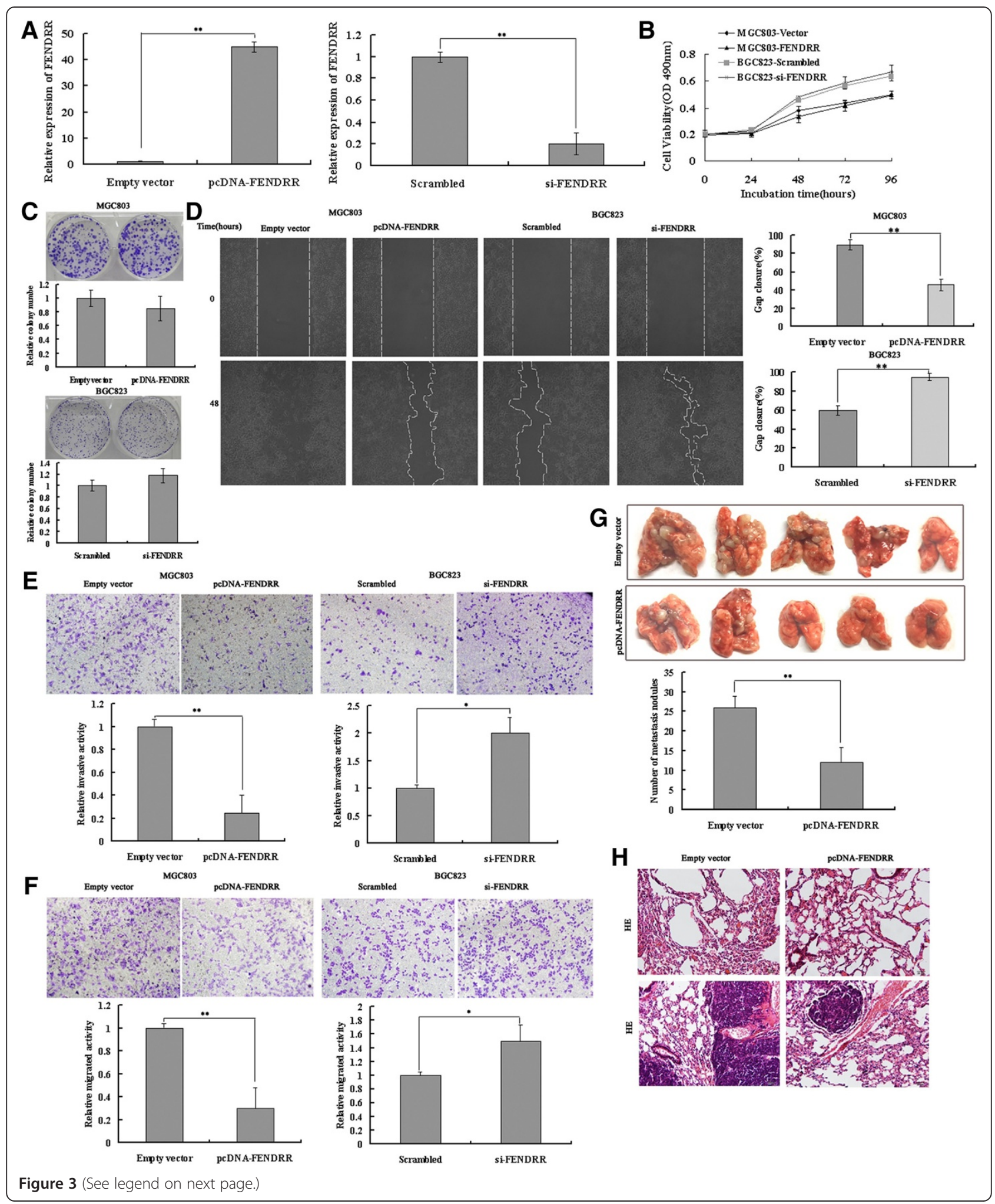




\section{(See figure on previous page.)}

Figure 3 Effects of FENDRR on gastric cancer cell migration and invasion in vitro and in vivo. MGC803 cells were transfected with pCDNA-FENDRR, and BGC823 cells were transfected with si-FENDRR. (A) qPCR analysis of FENDRR expression levels following the treatment of MGC803 cells with empty vector and pcDNA-FENDRR (left panel), and the treatment of BGC823 cells with scrambled siRNA and si-FENDRR (right panel). Experiments were performed in triplicate. Bars: SD; ${ }^{* *} p<0.01$. (B) Forty-eight hours after transfection, MTT assays were conducted to determine the proliferation of MGC803 and BGC823 cells. Experiments were performed in triplicate. Bars: SD. (C) Colony-formation growth assays were conducted to determine the proliferation of MGC803 and BGC823 cells. The colonies were counted and photographed. Experiments were performed in triplicate. Bars: SD. (D) Wound healing assays were used to investigate the migratory ability of gastric cancer cells. Experiments were performed in triplicate. Bars: SD; ${ }^{*} \mathrm{p}<0.01$. (E) and (F) Transwell assays were used to investigate the changes in the migratory and invasive abilities of gastric cancer cells. Experiments were performed in triplicate. Bars: SD; ${ }^{*} p<0.05$ and ${ }^{* *} p<0.01$. (G) MGC803 cells transfected with pcDNA-FENDRR and empty vector were separately injected into the tail veins of athymic mice. Lungs were harvested from the mice in each experimental group, and the numbers of tumor nodules visible on lung surfaces were counted. The assay was independently conducted twice. Bars: SD; ${ }^{*} p<0.05$ and ${ }^{* *} p<0.01$. (H) Visualization of the entire lung, and hematoxylin and eosin (HE)-stained lung sections.

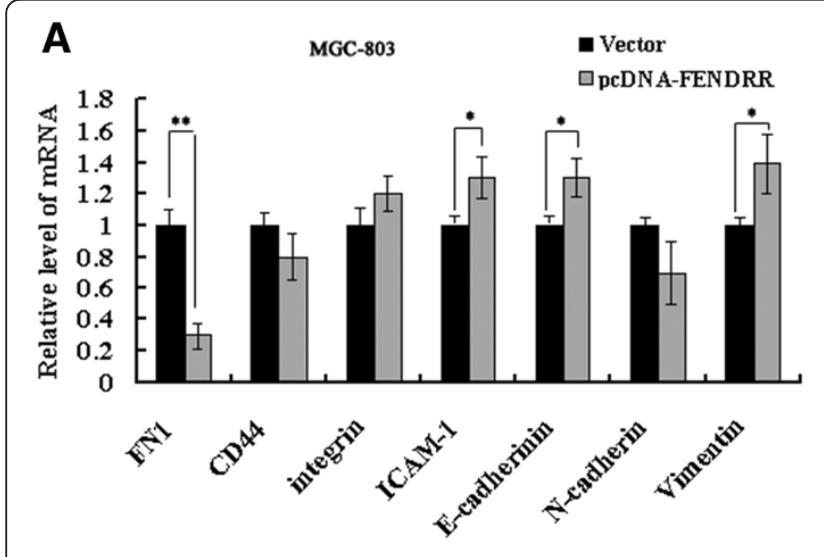

C


D
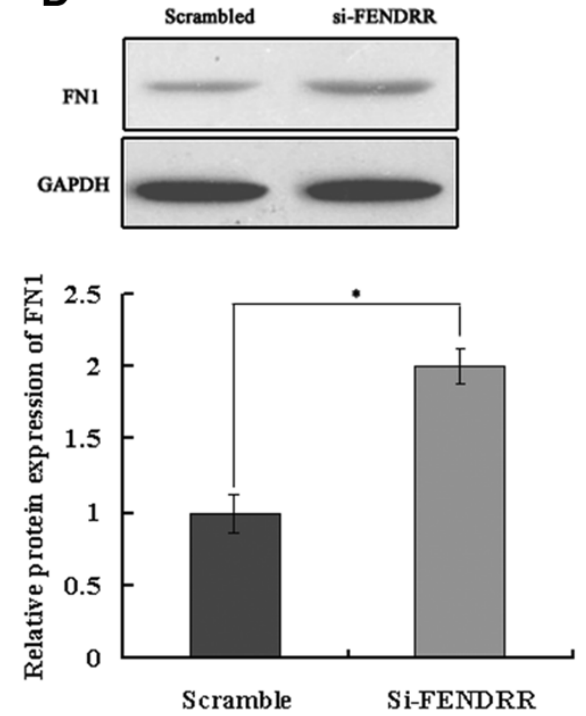



E

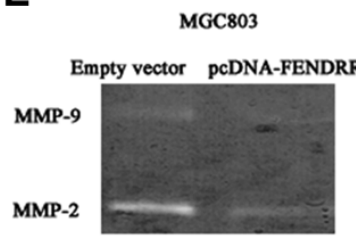

F $\quad$ BGC823

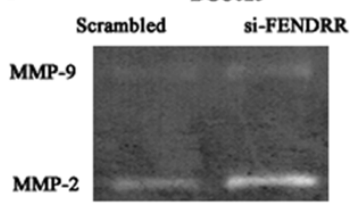

Figure 4 FENDRR regulates FN1 expression and MMP2/MMP9 activity. (A) Expression of cell adhesion molecules (FN1, integrin, CD44, ICAM-1, E-cadherin, N-cadherin and vimentin) as detected using qRT-PCR after FENDRR was overexpressed in MGC803 cells or (B) blocked in BGC823 cells. (C) After FENDRR is overexpressed in MGC803 cells, western blot analysis shows that the FN1 protein level is diminished, as compared to the level in the control group. (D) The FN1 protein level is elevated after FENDRR expression is blocked in BGC823 cells, as compared to the level in the control group. (E) and (F) FENDRR regulates the enzymatic activity of MMP2/MMP9 in gastric cancer cells. All the above experiments were performed in triplicate. Bars: SD; ${ }^{*} p<0.05$ and ${ }^{* *} p<0.01$. 


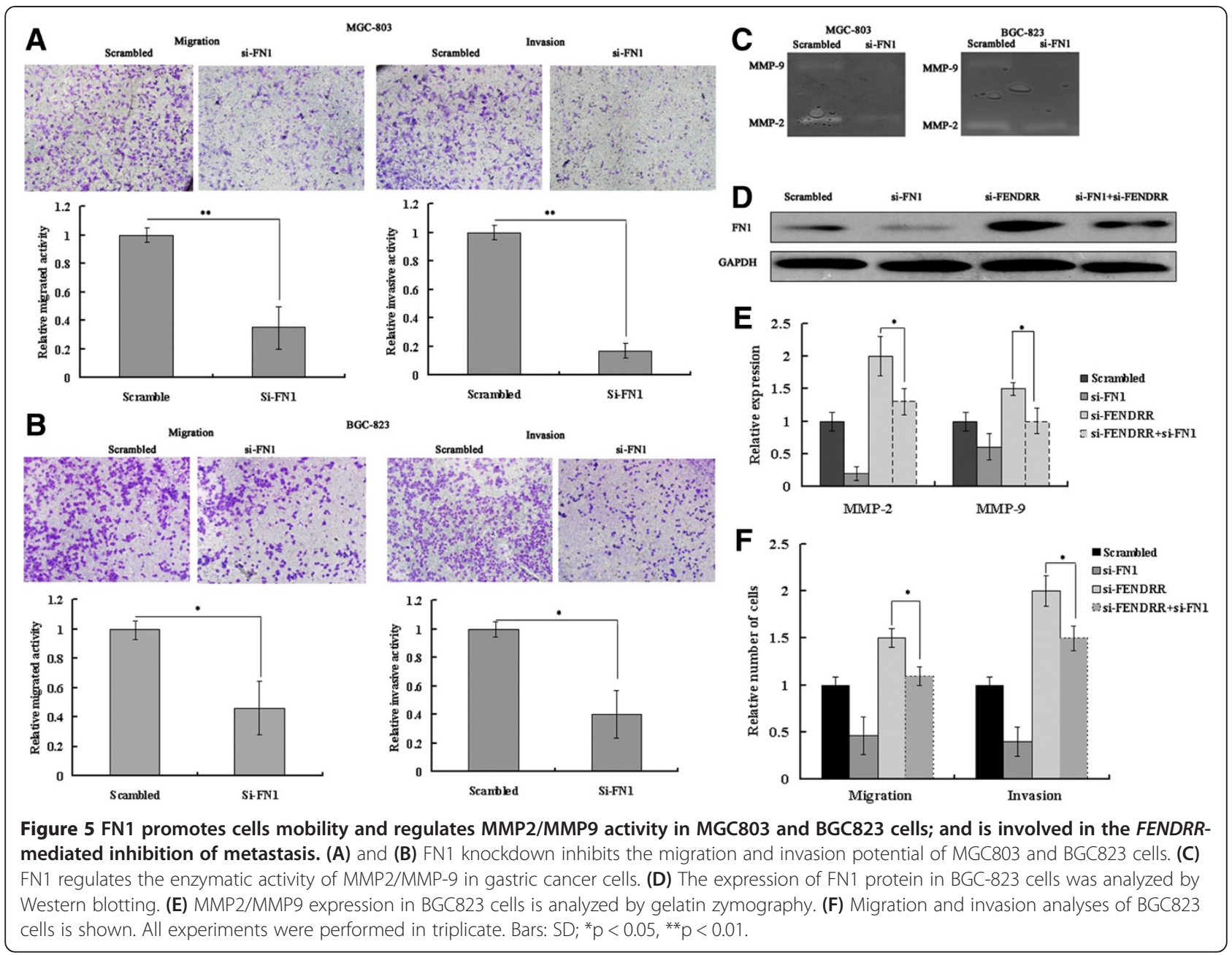

generally higher in gastric cancer tissues and cells, when compared with the levels in matched normal tissues and cells, respectively (Figure 6A,B).We also found that $80 \%$ of tumor-derived tissues showed higher FN1 protein levels, whereas most normal gastric tissues exhibited low FN1 protein levels, as compared with the levels in the paired tumor tissues (Figure 6C). Further analysis revealed that of FENDRR expression is inversely correlated with FN1 mRNA and protein levels in gastric cancer (Figure 6D, Additional file 4: Table S3). These data revealed that the FN1 level was mostly inversely correlated with the FENDRR level in gastric cancer.

\section{Discussion}

Khalil et al. first identified the lncRNA FENDRR and confirmed its PRC2-binding functions [17]. Subsequently, Grote et al. found that it is an essential regulator of heart and body wall development [15]. However, its function in carcinogenesis and tumor progression is unclear. In this study, we first detected that FENDRR levels were decreased in gastric cancer cells and tissues compared with normal gastric epithelial cells and adjacent normal tissues. Moreover, low expression of FENDRR was significantly correlated with aggressive tumor characteristics (greater invasion depth, higher tumor stage, and lymphatic metastasis) and poor prognosis; when the patients were subdivided into three groups according to tumor stage, we found that FENDRR expression could better distinguish patients with different outcomes in stage III and IV. However, we did not observe a significant correlation between FENDRR expression and clinical outcome in early clinical stages of gastric cancer (clinical stages I-II, p = 0.638 for DFS and 0.994 for OS), probably due to good prognosis in the early stage of gastric cancer and the limited number of patients with stage I-II gastric cancer. Univariate and multivariate analysis indicated that OS and DFS were significantly better among patients with high FENDRR expression than in patients with low $F E N D R R$ expression in the same stage. Multivariate analysis demonstrated that FENDRR expression was an independent prognostic factor for gastric cancer patients. 


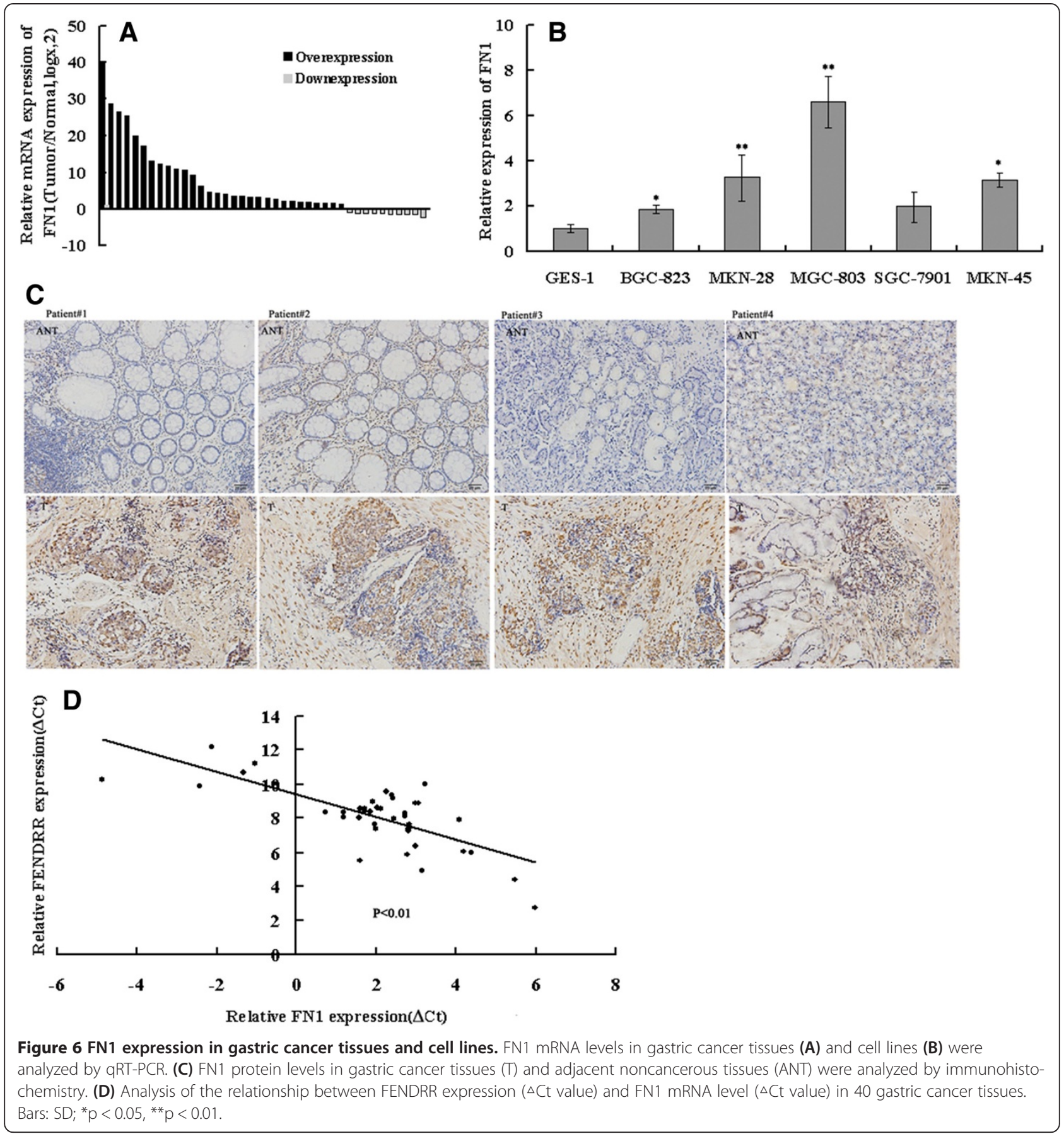

This suggests that FENDRR might be a promising prognostic biomarker in gastric cancer patients.

As low FENDRR expression was associated with an aggressive tumor phenotype in gastric cancer, we speculated that FENDRR could play a significant role in tumor biology. Initially, we chose representative cell lines of gastric cancer and investigated their FENDRR expression in comparison to a non-tumoral gastric cell line. We observed that 3 out of 5 tumoral cell lines exhibited low FENDRR expression, which corroborate our previous findings. We next determined whether FENDRR expression influenced tumor-like characteristics, such as migration, invasion, and metastasis. Indeed, ectopic expression of FENDRR inhibited cell migration and invasion, whereas knockdown of endogenous FENDRR expression significantly enhanced these capacities. Moreover, increased FENDRR expression significantly reduced the number of metastatic nodules on the lungs in vivo. However, no significant effect on cellular proliferation was observed after administration of 
ectopic expression or knockdown of FENDRR. This is in line with our clinical findings that FENDRR was significantly correlated with invasion depth, tumor stage, and lymphatic metastasis, but not tumor size. These results revealed that FENDRR might impact the prognosis of gastric cancer by affecting cell migration and invasion.

Many lncRNAs have been implicated in various types of cancers. Reportedly, the lncRNAs class MALAT-1 has been found to promote cell motility in lung adenocarcinoma cells [23]. PCGEM1 overexpression and PRNCR1 have been identified for their involvement in prostate carcinogens [24,25]. Gupta et al. [18] revealed that the IncRNA HOTAIR induces invasive and metastatic behavior in breast cancer cells. Tumor development and progression is precisely regulated by several subsets of genes that act by either silencing tumor suppressor genes or activating oncogenes [26]. In cancer cells, tumor-suppressor genes are usually silenced by genetic or epigenetic alterations [27]. Whether epigenetic regulatory factors, such as DNA methylation or histone acetylation, manipulate the expression of IncRNAs remains unkown. Hypermethylation of the promoter or the intergenic differentially methylated region has been found to contribute to reduced expression of lncRNA MEG3 in tumors, indicating that epigenetic regulation is also involved in the expression of these genes [28]. Our findings emphasize that histone acetylation is a key factor in controlling the expression of the lncRNA FENDRR. These results, along with those from a recent study [28], highlight the role of epigenetics in regulating lncRNA transcription.

To explore the molecular mechanism through which FENDRR contributes to invasion and metastasis in gastric cancer, we investigated potential target proteins involved in cell motility and matrix invasion. We identified which genes were differentially expressed upon the ectopic expression or depletion of FENDRR, in comparison with untreated cells, FN1 mRNA levels were reduced or elevated after overexpression or blocking of FENDRR, respectively. Western blot analysis was performed to confirm that FN1 protein levels were also regulated by FENDRR. Importantly, the expression and activity of MMP2/MMP9 were also reduced upon FENDRR overexpression. FN1 is an extracellularmatrix glycoprotein that plays major roles in cell differentiation, growth and migration. It is involved in processes such as wound healing and embryonic development, as well as oncogenic transformation [29]. Importantly, FN is a key mediator of disease progression and metastasis in diverse carcinomas, such as skin squamous cell carcinoma [20], brain glioblastoma [30], and laryngeal squamous carcinoma [31]. For instance, FN and tissue transglutaminase 2 (TG2) contribute to the metastatic activity of A431 tumor cells, and this mediation may be partly due to the enhancement of $\mathrm{FN}$ and $\beta$ integrin expression [20], FN1 is a key mediator of glioma progression through a mechanism that involves the maintenance of integrin $\beta 1 \mathrm{FN}$ receptors in glioma cells [32]. In this study, we found that FN1expression was upregulated in gastric cancer tissues and cell lines, when compared with the expression matched normal tissues and cells, respectively. We also confirmed that FN1 knockdown inhibits cell mobility in the gastric carcinoma cell line. Moreover, immunohistochemical analysis showed that the FN1 protein level was mostly inversely correlated with the FENDRR level in gastric cancer tissues, which indirectly confirmed that FN1 may be negatively regulated by FENDRR. MMPs are well known to play essential roles in invasion and metastasis in human carcinomas [33,34]. FN1 has been reported to activate MMP2/MMP9 to promote invasion and metastasis in multiple carcinomas $[21,22,35]$. We showed that cotransfection with si-FN1 and si-FENDRR could partly "rescue" the MMP2/MMP9 upregulation induced by FENDRR downregulation, indicating that FENDRR regulated MMP2/MMP9 activity partly through FN1. However, the precise molecular mechanisms how FENDRR regulated FN1and MMPs remains unclear and is required further investigation.

The above evidence shows that histone deacetylation downregulates FENDRR expression in gastric cancer, and decreased FENDRR expression induces FN1 expression. Subsequently, the increased FN1 expression contributes to the activation of MMP2/MMP9, leading to higher migration and invasion potential of gastric cancer cells, which was manifested by the greater number of metastatic nodules in the nude mice. Moreover, our data also identified that patients exhibiting low FENDRR expression have higher metastasis potential and poor clinical outcomes.

\section{Conclusion}

In summary, our study showed that FENDRR is dramatically downregulated in gastric cancer tissues and cell lines and that the low expression of FENDRR is significantly associated with invasion depth, tumor stage, lymphatic metastasis and patients' survival time. Moreover, upregulation of FENDRR has the effect of suppressing gastric cancer cell migration and invasion in vitro by targeting FN1 and MMP2/MMP9. Further insights into the functional and clinical implications of FENDRR and its targets FN1 and MMP2/MMP9 may help with the treatment of gastric cancer.

\section{Materials and methods Cell lines}

Human gastric adenocarcinoma cancer cell lines MGC803, BGC823, MKN28, MKN45 and SGC7901 and 
the normal gastric epithelium cell line (GES-1) were obtained from the Chinese Academy of Sciences Committee on Type Culture Collection cell bank (Shanghai, China). MGC803, BGC823 and MKN28 cells were cultured in RPMI 1640; MKN45, GES-1 and SGC7901 cells were cultured in DMEM (GIBCO-BRL) medium supplemented with $10 \%$ fetal bovine serum (FBS), $100 \mathrm{U} / \mathrm{ml}$ penicillin and $100 \mathrm{mg} / \mathrm{ml}$ streptomycin (Invitrogen, Carlsbad, CA, USA) at $37^{\circ} \mathrm{C}$ in $5 \% \mathrm{CO}_{2}$.

\section{Tissue samples and clinical data collection}

In this study, we analyzed 158 patients who underwent resection of the primary gastric cancer at the First Affiliated Hospital of Nanjing Medical University, Subei People's Hospital of Jiangsu Province, and Huai'an First People's Hospital of Jiangsu Province. The study was approved by the Ethics Committee on Human Research of the First Affiliated Hospital of Nanjing Medical University, Subei People's Hospital of Jiangsu Province and Huai'an First People's Hospital of Jiangsu Province and written informed consent was obtained from all patients. The clinicopathological characteristics of the gastric cancer patients are summarized in Table 1. All patients with gastric cancer have been followed up at intervals of 1-2 months until September 2013, and the median follow-up period was 36 months (range, 20-48 months). Follow-up studies included physical examination, laboratory analysis, and computed tomography if necessary. OS was defined as the interval between the dates of surgery and death. DFS was defined as the interval between the dates of surgery and recurrence; if recurrence was not diagnosed, patients were censored on the date of death or the last follow-up.

\section{RNA preparation and quantitative real-time PCR}

Total RNAs were extracted from tumorous and adjacent normal tissues or cultured cells using Trizol reagent (Invitrogen) following the manufacturer's protocol. RT and qPCR kits (Takara, Dalian, China) were used to evaluate the expression of FENDRR in tissue samples and cultured cells. The primers used in this study are shown in Additional file 5: Table S1. Real-time PCR was performed in triplicate, and the relative expression of FENDRR was calculated using the comparative cycle threshold (CT) $\left(2^{-\Delta \Delta C T}\right)$ method with glyceraldehyde-3phosphate dehydrogenase (GAPDH) as the endogenous control to normalize the data.

\section{Vector construction and transfection, and siRNA transfection}

To overexpress FENDRR, the coding sequence of FENDRR was amplified and subcloned into the pcDNA3.1 (+) vector (Invitrogen) according to the manufacturer' instructions. MGC803 cells were then transfected with a negative control vector or the FENDRR-expressing plasmid using Lipofectamine 2000 (Invitrogen). To generate FENDRRknockdown BGC823 cells, the target sequence for FENDRR siRNA or scrambled siRNA that did not correspond to any human sequence was synthesized by Invitrogen. To generate FN1-knockdown BGC823 and MGC803 cells, the target sequence for FN1 siRNA was transfected into the cells, using Lipofectamine 2000 (invitrogen), according to the manufacturer's instructions. The siRNAs si-HDAC1 and si-HDAC3 were transfected into BGC823 or MGC803 cells. The siRNA sequence were shown in Additional file 5: Table S1.

\section{Cell proliferation assays}

Cell viability was monitored using a Cell Proliferation Reagent Kit I (MTT) (Roche Applied Science). The BGC823 cells transfected with si-FENDRR (3000 cells/ well) and MGC803 cells transfected with pCDNAFENDRR were grown in 96-well plates. Cell viability was assessed every $24 \mathrm{~h}$ following the manufacturer's protocol. All experiments were performed in quadruplicate. For colony formation assays, pCDNA-FENDRR-transfected MGC803 cells $(n=500)$ were placed in a 6-well plates and maintained in media containing 10\% FBS. The medium was replaced every 4 days; after 14 days, the cells were fixed with methanol and stained with $0.1 \%$ crystal violet (Sigma-Aldrich). Visible colonies were then counted. For each treatment group, wells were assessed in triplicate, and experiments were independently repeated three times.

\section{Wound healing assay}

For the wound healing assay, $3 \times 10^{5}$ cells were seeded in 6-well plates, cultured overnight, and transfected with pCDNA-FENDRR, si-FENDRR or a control. Once cultures reached $85 \%$ confluence, the cell layer was scratched with a sterile plastic tip and washed with culture medium. The cells were then cultured for $48 \mathrm{~h}$ with medium containing $1 \%$ FBS. At different time points, images of the plates were acquired using a microscope. The distance between the two edges of the scratch was measured using the Digimizer software system. The assay was independently repeated three times.

\section{Cell migration and invasion assays}

For the migration assays, at $48 \mathrm{~h}$ post-transfection, $5 \times 10^{4}$ cells in serum-free media were placed into the upper chamber of an insert ( $8-\mu \mathrm{m}$ pore size; Millipore). For the invasion assays, $1 \times 10^{5}$ cells in serum-free medium were placed into the upper chamber of an insert coated with Matrigel (Sigma-Aldrich). Medium containing 10\% FBS was added to the lower chamber. After incubation for $24 \mathrm{~h}$, the cells remaining on the upper membrane were removed with cotton wool. Cells that had migrated or 
invaded through the membrane were stained with methanol and $0.1 \%$ crystal violet, imaged, and counted using an IX71 inverted microscope (Olympus, Tokyo, Japan). Experiments were independently repeated three times.

\section{Western blot assay and antibodies}

Cells were lysed using RIPA protein extraction reagent (Beyotime, Beijing, China) supplemented with a protease inhibitor cocktail (Roche, CA, USA) and phenylmethylsulfonyl fluoride (Roche). The concentration of proteins was determined using a Bio-Rad protein assay kit. Protein extracts $(50 \mu \mathrm{g})$ were separated by $10 \%$ sodium dodecyl sulfate-polyacrylamide gel electrophoresis (SDS-PAGE), transferred to nitrocellulose membranes (Sigma) and incubated with specific antibodies. Electrochemiluminescence (ECL) chromogenic substrate was used to visualize the bands and the intensity of the bands was quantified by densitometry (Quantity One software; Bio-Rad), with GAPDH used as a control. Antibodies (1:1000 dilution) against FN1 were purchased from BD.

\section{Gelatin zymography}

Conditioned media were obtained after a 30-h incubation of the different BGC823 and MGC803 cells in serum-free medium and were then concentrated 80-fold using Amicon Ultra Centrifugal Filter Units (Millipore) and normalized by protein concentration. Samples were loaded on $10 \%$ SDS-PAGE gels containing $0.1 \%$ gelatin. Electrophoresis was carried out under nonreducing conditions at $100 \mathrm{~V}$ and $4^{\circ} \mathrm{C}$. The gels were then washed in $2.5 \%$ Triton $\mathrm{X}-100$, incubated in substrate buffer $(50 \mathrm{mmol} / \mathrm{L}$ Tris$\mathrm{HCl}, \mathrm{pH}$ 8.0; $50 \mathrm{mmol} / \mathrm{L} \mathrm{NaCl} ; 10 \mathrm{mmol} / \mathrm{L} \mathrm{CaCl}$; and $0.05 \%$ Brij 35) for $40 \mathrm{~h}$ at $37^{\circ} \mathrm{C}$, stained with Coomassie stain solution (Bio-Rad), and destained in $20 \%$ methanol and $10 \%$ acetic acid. Gelatinolytic activity was identified as a clear band on a blue background. The activities of secreted MMPs were detected using gelatin zymography as previously described [36], with several modifications.

\section{Tail vein injections into athymic mice}

Male athymic mice (4-weeks-old) were purchased from the Animal Center of the Chinese Academy of Science (Shanghai, China) and maintained in laminar flow cabinets under specific pathogen-free conditions. BGC823 cells transfected with pCDNA-FENDRR or the empty vector were harvested from 6-well plates, washed with phosphate-buffered saline (PBS), and resuspended at a density of $2 \times 10^{7}$ cells $/ \mathrm{ml}$. The cell suspension $(0.1 \mathrm{ml})$ was injected into the tail veins of 10 mice, which were sacrificed 7 weeks after the injection. The lungs were removed and photographed, and visible tumors on the lung surface were counted. This study was carried out in strict accordance with the Guide for the Care and Use of Laboratory Animals of the National Institutes of
Health. Our protocol was approved by the Committee on the Ethics of Animal Experiments of Nanjing Medical University (Permit Number: 200933). All surgery was performed under sodium pentobarbital anesthesia, and all efforts were made to minimize suffering [37]. The assays were independently performed for two replicates.

\section{Immunohistochemical (IHC) analysis}

The immunohistochemical analysis of FN1 was performed according to a previously described method [38]. To quantify FN1 protein expression, both the intensity and extent of immunoreactivity were evaluated and scored. In the present study, staining intensity was scored as follows: 0, negative staining; 1, weak staining; 2, moderate staining; and 3, strong staining. The scores of the extent of immunoreactivity ranged from 0 to 3 , and were determined according to the percentage of cells that showed positive staining in each microscopic field of view $(0,<25 \% ; 1,25 \%-50 \% ; 2,50 \%-75 \% ; 3$, $75 \%-100 \%)$. A final score ranging from 0 to 9 was achieved by multiplying the scores for intensity and extent.

\section{Statistical analysis}

All statistical analyses were performed using SPSS 20.0 software (IBM, SPSS, Chicago, IL, USA). The significance of the differences between groups was estimated by the Student t-test, $\chi^{2}$ test, or Wilcoxon test, as appropriate. DFS and OS rates were calculated by the Kaplan-Meier method with the log-rank test applied for comparison. Survival data were evaluated using univariate and multivariate Cox proportional hazards models. Variables with a value of $\mathrm{p}<0.05$ in univariate analysis were used in subsequent multivariate analysis on the basis of Cox regression analyses. Kendall's Tau-b and Pearson correlation analyses were performed to investigate the correlation between FENDRR and FN1 protein expressions. Two-sided p-values were calculated, and a probability level of 0.05 was chosen for statistical significance.

\section{Additional files}

Additional file 1: Figure S1. qPCR analysis of HDAC1 and HDAC3 expression levels following the treatment of BGC823 and MGC803 cells with si-HDAC1 and si-HDAC3. Bars: SD; ${ }^{*} p<0.05$, ${ }^{* *} p<0.01$.

Additional file 2: Table S2. Clinical data of all patients involved in the study.

Additional file 3: Figure S2. MGC803 and BGC823 cells were transfected with an siRNA targeting FN1, causing the suppression of the mRNA (A) and protein levels (B) of FN1. Bars: SD; ${ }^{*} p<0.05,{ }^{* *} p<0.01$.

Additional file 4: Table S3. Relative FENDRR expression level and FN1 scores on immunohistochemistry. 
Additional file 5: Table S1. Primers used for $q R T-P C R$ and siRNA oligonucleotides.

\section{Abbreviations}

IncRNA: Long non-coding RNA; HDAC: Histone deacetylase; TSA: Trichostatin A; GC: Gastric cancer; DFS: Disease-free survival; OS: Overall survival; HR: Hazard ratio; FN1: Fibronectin; MMPs: Matrix metalloproteases.

\section{Competing interests}

The authors declare that they have no competing interests.

\section{Authors' contributions}

XTP designed the study, detected the cells biological function, conducted the GRT-PCR assays, carried out the Western blotting assays, established the animal model, performed the statistical analysis, performed the immunohistochemistry assays, and drafted the manuscript. HMD and LXX provided the tissue samples and the clinical data, XR participated in the design of the study, and administrated the Gelatin zymography assays, SM, CWM, HL, YL, ZEB, KR, and DW helped to acquire experimental data. SYQ conceived the study, participated in its design and coordination, and helped to draft the manuscript. All authors read and approved the final manuscript.

\section{Acknowledgements}

This study was supported by the National Natural Science Foundation of China (81172140, 81272532), Jiangsu Province Clinical Science and Technology projects (Clinical Research Center, BL2012008) and the Priority Academic Program Development of Jiangsu Higher Education Institutions (Public Health and Preventive Medicine, JX10231801).

\section{Author details}

${ }^{1}$ Department of Oncology, the First Affiliated Hospital of Nanjing Medical University, No. 300 Guangzhou Road, Nanjing 210029, Jiangsu Province, People's Republic of China. ${ }^{2}$ Department of Medical Oncology, Huai'an First People's Hospital, Nanjing Medical University, No. 6 Beijing Road West, Huai'an 223300Jiangsu Province, People's Republic of China. ${ }^{3}$ Department of Biochemistry and Molecular Biology, Nanjing Medical University, Nanjing, Jiangsu Province, People's Republic of China. ${ }^{4}$ Department of Gastrointestinal Surgery, Subei People's Hospital of Jiangsu Province, Yangzhou University, Yangzhou 225001Jiangsu Province, People's Republic of China.

Received: 9 May 2014 Accepted: 19 August 2014

Published: 29 August 2014

\section{References}

1. Moore MA, Attasara P, Khuhaprema T, Le TN, Nguyen TH, Raingsey PP, Sriamporn S, Sriplung H, Srivanatanakul P, Bui DT, Wiangnon S, Sobue T: Cancer epidemiology in mainland South-East Asia - past, present and future. Asian Pac J Cancer Prev 2010, 11(Suppl 2):67-80

2. Jemal A, Siegel $R, X u$ J, Ward E: Cancer statistics, 2010. CA Cancer J Clin 2010, 60:277-300.

3. Wang $\mathrm{XN}$, Liang $\mathrm{H}$ : Some problems in the surgical treatment of gastric cancer. Chin J Cancer 2010, 29:369-373.

4. Steeg PS: Metastasis suppressors alter the signal transduction of cancer cells. Nat Rev Cancer 2003, 3:55-63.

5. Milne AN, Carneiro F, O'Morain C, Offerhaus GJ: Nature meets nurture: molecular genetics of gastric cancer. Hum Genet 2009, 126:615-628.

6. Ponting CP, Belgard TG: Transcribed dark matter: meaning or myth? Hum Mol Genet 2010, 19:R162-R168.

7. Muers M: RNA: Genome-wide views of long non-coding RNAs. Nat Rev Genet 2011, 12:742.

8. Ponting $\mathrm{CP}$, Oliver $\mathrm{PL}$, Reik W: Evolution and functions of long noncoding RNAs. Cell 2009, 136:629-641.

9. Rinn JL, Kertesz M, Wang JK, Squazzo SL, Xu X, Brugmann SA, Goodnough $L H$, Helms JA, Farnham PJ, Segal E, Chang HY: Functional demarcation of active and silent chromatin domains in human HOX loci by noncoding RNAs. Cell 2007, 129:1311-1323.

10. Pibouin L, Villaudy J, Ferbus D, Muleris M, Prosperi MT, Remvikos Y, Goubin $\mathrm{G}$ : Cloning of the mRNA of overexpression in colon carcinoma-1: a sequence overexpressed in a subset of colon carcinomas. Cancer Genet Cytogenet 2002, 133:55-60.
11. Fu X, Ravindranath L, Tran N, Petrovics G, Srivastava S: Regulation of apoptosis by a prostate-specific and prostate cancer-associated noncoding gene, PCGEM1. DNA Cell Biol 2006, 25:135-141.

12. Calin GA, Liu CG, Ferracin M, Hyslop T, Spizzo R, Sevignani C, Fabbri M, Cimmino A, Lee EJ, Wojcik SE, Shimizu M, Tili E, Rossi S, Taccioli C, Pichiorri F, Liu X, Zupo S, Herlea V, Gramantieri L, Lanza G, Alder H, Rassenti L, Volinia S, Schmittgen TD, Kipps TJ, Negrini M, Croce CM: Ultraconserved regions encoding ncRNAs are altered in human leukemias and carcinomas. Cancer Cell 2007, 12:215-229.

13. Lin R, Maeda S, Liu C, Karin M, Edgington TS: A large noncoding RNA is a marker for murine hepatocellular carcinomas and a spectrum of human carcinomas. Oncogene 2007, 26:851-858.

14. Qi P, Du X: The long non-coding RNAs, a new cancer diagnostic and therapeutic gold mine. Mod Pathol 2013, 26:155-165.

15. Grote P, Wittler L, Hendrix D, Koch F, Wahrisch S, Beisaw A, Macura K, Blass $G$, Kellis M, Werber M, Herrmann BG: The tissue-specific IncRNA Fendrr is an essential regulator of heart and body wall development in the mouse. Dev Cell 2013, 24:206-214.

16. Schuettengruber B, Chourrout D, Vervoort M, Leblanc B, Cavalli G: Genome regulation by polycomb and trithorax proteins. Cell 2007, 128:735-745.

17. Khalil AM, Guttman M, Huarte M, Garber M, Raj A, Rivea Morales D, Thomas K, Presser A, Bernstein BE, Van Oudenaarden A, Regev A, Lander ES, Rinn JL: Many human large intergenic noncoding RNAs associate with chromatin-modifying complexes and affect gene expression. Proc Nat Acad Sci U S A 2009, 106:11667-11672.

18. Gupta RA, Shah N, Wang KC, Kim J, Horlings HM, Wong DJ, Tsai MC, Hung T, Argani P, Rinn JL, Wang Y, Brzoska P, Kong B, Li R, West RB, van de Vijver MJ, Sukumar S, Chang HY: Long non-coding RNA HOTAIR reprograms chromatin state to promote cancer metastasis. Nature 2010, 464:1071-1076.

19. Nilsson J, Helou K, Kovacs A, Bendahl PO, Bjursell G, Ferno M, Carlsson P, Kannius-Janson M: Nuclear Janus-activated kinase 2/nuclear factor 1-C2 suppresses tumorigenesis and epithelial-to-mesenchymal transition by repressing Forkhead box F1. Cancer Res 2010, 70:2020-2029.

20. Chen SH, Lin CY, Lee LT, Chang GD, Lee PP, Hung CC, Kao WT, Tsai PH, Schally $A V$, Hwang JJ, Lee MT: Up-regulation of fibronectin and tissue transglutaminase promotes cell invasion involving increased association with integrin and MMP expression in A431 cells. Anticancer Res 2010, 30:4177-4186.

21. Sen T, Dutta A, Maity G, Chatterjee A: Fibronectin induces matrix metalloproteinase-9 (MMP-9) in human laryngeal carcinoma cells by involving multiple signaling pathways. Biochimie 2010, 92:1422-1434.

22. Moroz A, Delella FK, Lacorte LM, Deffune E, Felisbino SL: Fibronectin induces MMP2 expression in human prostate cancer cells. Biochem Biophys Res Commun 2013, 430:1319-1321.

23. Tano K, Mizuno R, Okada T, Rakwal R, Shibato J, Masuo Y, ljiri K, Akimitsu N MALAT-1 enhances cell motility of lung adenocarcinoma cells by influencing the expression of motility-related genes. FEBS Lett 2010, 584:4575-4580.

24. Srikantan V, Zou Z, Petrovics G, Xu L, Augustus M, Davis L, Livezey JR, Connell T, Sesterhenn IA, Yoshino K, Buzard GS, Mostofi FK, McLeod DG, Moul JW, Srivastava S: PCGEM1, a prostate-specific gene, is overexpressed in prostate cancer. Proc Natl Acad Sci U S A 2000, 97:12216-12221.

25. Chung S, Nakagawa H, Uemura M, Piao L, Ashikawa K, Hosono N, Takata R, Akamatsu S, Kawaguchi T, Morizono T, Tsunoda T, Daigo Y, Matsuda K, Kamatani N, Nakamura Y, Kubo M: Association of a novel long non-coding RNA in 8q24 with prostate cancer susceptibility. Cancer Sci 2011, 102:245-252.

26. Stanbridge EJ: Identifying tumor suppressor genes in human colorectal cancer. Science 1990, 247:12-13.

27. Kanwal R, Gupta S: Epigenetic modifications in cancer. Clin Genet 2012, 81:303-311.

28. Benetatos L, Dasoula A, Hatzimichael E, Georgiou I, Syrrou M, Bourantas KL: Promoter hypermethylation of the MEG3 (DLK1/MEG3) imprinted gene in multiple myeloma. Clin Lymphoma Myeloma 2008, 8:171-175.

29. Pankov R, Yamada KM: Fibronectin at a glance. J Cell Sci 2002, 115:3861-3863.

30. Ritzenthaler JD, Han S, Roman J: Stimulation of lung carcinoma cell growth by fibronectin-integrin signalling. Mol Biosyst 2008, 4:1160-1169.

31. Wang F, Song G, Liu M, Li X, Tang H: miRNA-1 targets fibronectin1 and suppresses the migration and invasion of the HEp2 laryngeal squamous carcinoma cell line. FEBS Lett 2011, 585:3263-3269.

32. Sengupta S, Nandi S, Hindi ES, Wainwright DA, Han Y, Lesniak MS: Short hairpin RNA-mediated fibronectin knockdown delays tumor growth in a mouse glioma model. Neoplasia 2010, 12:837-847. 
33. Dong QZ, Wang Y, Tang ZP, Fu L, Li QC, Wang ED, Wang EH: Derlin-1 is overexpressed in non-small cell lung cancer and promotes cancer cell invasion via EGFR-ERK-mediated up-regulation of MMP-2 and MMP-9. Am J Pathol 2013, 182:954-964.

34. Katayama A, Bandoh N, Kishibe K, Takahara M, Ogino T, Nonaka S,

Harabuchi Y: Expressions of matrix metalloproteinases in early-stage oral squamous cell carcinoma as predictive indicators for tumor metastases and prognosis. Clin Cancer Res 2004, 10:634-640.

35. Qian P, Zuo Z, Wu Z, Meng X, Li G, Wu Z, Zhang W, Tan S, Pandey V, Yao Y, Wang P, Zhao L, Wang J, Wu Q, Song E, Lobie PE, Yin Z, Zhu T: Pivotal role of reduced let-7 g expression in breast cancer invasion and metastasis. Cancer Res 2011, 71:6463-6474.

36. Menshikov M, Elizarova E, Plakida K, Timofeeva A, Khaspekov G, Beabealashvilli R, Bobik A, Tkachuk V: Urokinase upregulates matrix metalloproteinase-9 expression in THP-1 monocytes via gene transcription and protein synthesis. Biochem J 2002, 367:833-839.

37. Kilkenny C, Browne W, Cuthill IC, Emerson M, Altman DG, Group NCRRGW, Altman D, Balding D, Cuthill I, Dunn C, Emerson M, Hurtley S, McGrath I, Stanford C: Animal research: reporting in vivo experiments: the ARRIVE guidelines. J Gene Med 2010, 12:561-563.

38. Sun M, Liu XH, Li JH, Yang JS, Zhang EB, Yin DD, Liu ZL, Zhou J, Ding Y, Li SQ, Wang ZX, Cao XF, De W: MiR-196a is upregulated in gastric cancer and promotes cell proliferation by downregulating p27(kip1). Mol Cancer Ther 2012, 11:842-852.

doi:10.1186/s13045-014-0063-7

Cite this article as: Xu et al:: Decreased expression of the long non-coding RNA FENDRR is associated with poor prognosis in gastric cancer and FENDRR regulates gastric cancer cell metastasis by affecting fibronectin1 expression. Journal of Hematology \& Oncology 2014 7:63.

\section{Submit your next manuscript to BioMed Central and take full advantage of:}

- Convenient online submission

- Thorough peer review

- No space constraints or color figure charges

- Immediate publication on acceptance

- Inclusion in PubMed, CAS, Scopus and Google Scholar

- Research which is freely available for redistribution 